\title{
Nonexistence of perfect permutation codes under the Kendall $\tau$-metric
}

\author{
Xiang Wang ${ }^{1}$ Wang Yuanjie ${ }^{1}$ Yin Wenjuan ${ }^{2}{ }^{*}$ Fang-Wei Fu $^{2}$ \\ ${ }^{1}$ National Computer Network Emergency Response Technical Team/Coordination Center of China (CNCERT/CC) \\ ${ }^{2}$ Chern Institute of Mathematics and LPMC, Nankai University, Tianjin 300071, China \\ E-mail: xqwang@mail.nankai.edu.cn,wang.yuanjie@outlook.com, ywjaimama@163.com,fwfu@nankai.edu.cn
}

\begin{abstract}
In the rank modulation scheme for flash memories, permutation codes have been studied. In this paper, we study perfect permutation codes in $S_{n}$, the set of all permutations on $n$ elements, under the Kendall $\tau$-Metric. We answer one open problem proposed by Buzaglo and Etzion. That is, proving the nonexistence of perfect codes in $S_{n}$, under the Kendall $\tau$-metric, for more values of $n$. Specifically, we present the recursive formulas for the size of a ball with radius $r$ in $S_{n}$ under the Kendall $\tau$-metric. Further, We prove that there are no perfect $t$-error-correcting codes in $S_{n}$ under the Kendall $\tau$-metric for some $n$ and $t=2,3,4$, or 5 .
\end{abstract}

Keywords: Flash memory, Perfect codes, Kendall $\tau$-Metric, Permutation codes.

\section{Introduction}

Flash memory is a non-volatile storage medium that is both electrically programmable and erasable. The rank modulation scheme for flash memories has been proposed in [2]. In this scheme, one permutation corresponds to a relative ranking of all the flash memory cells' levels. A permutation code is a nonempty subset of $S_{n}$, where $S_{n}$ is the set of all the permutations over $\{1,2, \ldots, n\}$. Permutation codes have been studied under various metrics, such as the $\ell_{\infty}$-metric $[4,6,7]$, the Ulam metric [11], and the Kendall $\tau$-metric $[3,5,8,9]$.

In this paper, we will focus on permutation codes under the Kendall $\tau$-metric. The Kendall $\tau$-distance [7] between two permutations $\pi, \sigma \in S_{n}$ is the minimum number of adjacent transpositions required to obtain the permutation $\sigma$ from $\pi$, where an adjacent transposition is an exchange of two distinct adjacent elements. Permutation codes under

\footnotetext{
*Corresponding Author
} 
the Kendall $\tau$-distance with minimum distance $d$ can correct up to $\left\lfloor\frac{d-1}{2}\right\rfloor$ errors. Let $A(n, d)$ be the size of the largest code in $S_{n}$ with minimum Kendall $\tau$-distance $d$. The bounds on $A(n, d)$ were proposed in $[3,10,14,15]$. Some $t$-error-correcting codes in $S_{n}$ were constructed in $[1,3,8,12,13]$. Buzaglo and Etzion [10] proved that there are no perfect single-error-correcting codes in $S_{n}$, where $n>4$ is a prime or $4 \leq n \leq 10$. They further [10] proposed the open problem to prove the nonexistence of perfect codes in $S_{n}$, under the Kendall $\tau$-metric, for more values of $n$ and/or other distances. In this paper, we prove that there are no perfect $t$-error-correcting codes in $S_{n}$ under the Kendall $\tau$ metric for some $n$ and $t=2,3,4$, or 5 . Specially, we prove that there are no perfect two-error-correcting codes in $S_{n}$, where $n+2>6$ is a prime. We also prove that there are no perfect three-error-correcting codes in $S_{n}$, where $n+1>6$ is a prime, $n^{2}+2 n-6$ has a prime factor $p>n$, or $4 \leq n \leq 33$. We further prove that there are no perfect four-error-correcting codes in $S_{n}$, where $n+1>6$ or $n+2>7$ is a prime, $n^{2}+3 n-12$ has a prime factor $p>n$, or $5 \leq n \leq 19$. Finally, we prove that there are no perfect five-error-correcting codes in $S_{n}$, where $n+7 \geq 12$ is a prime or $n^{3}+3 n^{2}-6 n-28$ has a prime factor $p>n$.

The rest of this paper is organized as follows. In Section 2, we will give some basic definitions for the Kendall $\tau$-metric and for perfect permutation codes. In Section 3, we determine the size of some balls with radius $r$ in $S_{n}$ under the Kendall $\tau$-metric. In Section 4 , we prove the nonexistence of a perfect $t$-error-correcting code in $S_{n}$ for some $n$ and $t=2,3,4$, or 5 by using the sphere packing upper bound. Section ?? concludes this paper.

\section{Preliminaries}

In this section we give some definitions and notations for the Kendall $\tau$-metric and perfect permutation codes. In addition, we summarize some important known facts.

Let $[n]$ denote the set $\{1,2, \ldots, n\}$. Let $S_{n}$ be the set of all the permutations over $[n]$. We denote by $\pi \triangleq[\pi(1), \pi(2), \ldots, \pi(n)]$ a permutation over $[n]$. For two permutations $\sigma, \pi \in S_{n}$, their multiplication $\pi \circ \sigma$ is denoted by the composition of $\sigma$ on $\pi$, i.e., $\pi \circ \sigma(i)=\sigma(\pi(i))$, for all $i \in[n]$. Under this operation, $S_{n}$ is a noncommutative group of size $\left|S_{n}\right|=n$ !. Denote by $\epsilon_{n} \triangleq[1,2, \ldots, n]$ the identity permutation of $S_{n}$. Let $\pi^{-1}$ be the inverse element of $\pi$, for any $\pi \in S_{n}$. For an unordered pair of distinct numbers $i, j \in[n]$, this pair forms an inversion in a permutation $\pi$ if $i<j$ and simultaneously $\pi(i)>\pi(j)$.

Given a permutation $\pi=[\pi(1), \pi(2), \ldots, \pi(i), \pi(i+1), \ldots \pi(n)] \in S_{n}$, an adjacent transposition is an exchange of two adjacent elements $\pi(i), \pi(i+1)$, resulting in the permutation $[\pi(1), \pi(2), \ldots, \pi(i+1), \pi(i), \ldots \pi(n)]$ for some $1 \leq i \leq n-1$. For any two permutations $\sigma, \pi \in S_{n}$, the Kendall $\tau$-distance between two permutations $\pi, \sigma$, denoted by $d_{K}(\pi, \sigma)$, is the minimum number of adjacent transpositions required to obtain the permutation $\sigma$ from $\pi$. The expression for $d_{K}(\pi, \sigma)[3]$ is as follows:

$$
d_{K}(\sigma, \pi)=\left|\left\{(i, j): \sigma^{-1}(i)<\sigma^{-1}(j) \wedge \pi^{-1}(i)>\pi^{-1}(j)\right\}\right| .
$$

For $\pi \in S_{n}$, the Kendall $\tau$-weight of $\pi$, denoted by $w_{K}(\pi)$, is defined as the Kendall 
$\tau$-distance between $\pi$ and the identity permutation $\epsilon_{n}$. Clearly, $w_{K}(\pi)$ is the number of inversions in the permutation $\pi$.

Definition 1. For $1 \leq d \leq\left(\begin{array}{c}n \\ 2\end{array}\right), C \subset S_{n}$ is an $(n, d)$-permutation code under the Kendall $\tau$-metric, if $d_{K}(\sigma, \pi) \geq d$ for any two distinct permutations $\pi, \sigma \in C$.

For a permutation $\pi \in S_{n}$, the Kendall $\tau$-ball of radius $r$ centered at $\pi$, denoted as $B_{K}^{n}(\pi, r)$, is defined by $B_{K}^{n}(\pi, r) \triangleq\left\{\sigma \in S_{n} \mid d_{K}(\sigma, \pi) \leq r\right\}$. For a permutation $\pi \in S_{n}$, the Kendall $\tau$-sphere of radius $r$ centered at $\pi$, denoted as $S_{K}^{n}(\pi, r)$, is defined by $S_{K}^{n}(\pi, r) \triangleq$ $\left\{\sigma \in S_{n} \mid d_{K}(\sigma, \pi)=r\right\}$. The size of a Kendall $\tau$-ball or a $\tau$-sphere of radius $r$ does not depend on the center of the ball under the Kendall $\tau$-metric. Thus, we denote the size of $B_{K}^{n}(\pi, r)$ and $S_{K}^{n}(\pi, r)$ as $B_{K}^{n}(r)$ and $S_{K}^{n}(r)$, respectively. We denote the largest size of an $(n, d)$-permutation code under the Kendall $\tau$-metric as $A_{K}(n, d)$. The sphere-packing bound for permutation codes under the Kendall $\tau$-metric are as follows:

Proposition 1. [3, Theorems 17 and 18]

$$
A_{K}(n, d) \leq \frac{n !}{B_{K}^{n}\left(\left\lfloor\frac{d-1}{2}\right\rfloor\right)} .
$$

When $d=2 r+1$, an $(n, 2 r+1)$-permutation code $C$ under the Kendall $\tau$-metric is called a perfect permutation code under the Kendall $\tau$-metric if it attains the spherepacking bound, i.e., $|C| \cdot B_{K}^{n}(r)=n$ !. That is, the balls with radius $r$ centered at the codewords of $C$ form a partition of $S_{n}$. A perfect $(n, 2 r+1)$-permutation code under the Kendall $\tau$-metric is also called a perfect $r$-error-correcting code under the Kendall $\tau$-metric.

In [10], Buzaglo and Etzion proved that there does not exist a perfect one-errorcorrecting code under the Kendall $\tau$-metric if $n>4$ is a prime or $4 \leq n \leq 10$. Based on the above definitions and notations, we will prove the nonexistence of a perfect $t$-errorcorrecting code in $S_{n}$ under the Kendall $\tau$-metric for some $n$ and $t=2,3,4$, or 5 by using the sphere-packing upper bound in the following sections.

\section{The size of a ball or a sphere with radius $r$ in $S_{n}$ under the Kendall $\tau$-metric}

In this section, we compute the size of a ball or a sphere with radius $r$ in $S_{n}$ under the Kendall $\tau$-metric and give recursive formulas of $B_{K}^{n}(r)$ and $S_{K}^{n}(r)$, respectively. Since $B_{K}^{n}(r)$ does not depend on the center of the ball, we consider the ball $B_{K}^{n}\left(\epsilon_{n}, r\right)$ which is a ball with radius $r$ centered at the identity permutation $\epsilon_{n}$ and denote by $S_{K}^{n}\left(\epsilon_{n}, r\right) \triangleq$ $\left\{\sigma \in S_{n} \mid d_{K}\left(\sigma, \epsilon_{n}\right)=w_{k}(\sigma)=r\right\}$ the sphere centered at $\epsilon_{n}$ and of radius $r$.

\subsection{The size of a sphere of radius $r$ in $S_{n}$ under the Kendall $\tau$-metric}

In order to give the property of $S_{K}^{n}(r)$, we require some notations and lemmas in [10]. For a permutation $\pi=[\pi(1), \pi(2), \ldots, \pi(n)] \in S_{n}$, the reverse of $\pi$ is the permutation 
$\pi^{r} \triangleq[\pi(n), \pi(n-1), \ldots, \pi(2), \pi(1)]$. For all $\pi \in S_{n}$, we have $w_{K}(\pi) \leq\left(\begin{array}{l}n \\ 2\end{array}\right)$. For convenience, we denote $S_{K}^{n}(r)=0$ for $r \geq\left(\begin{array}{l}n \\ 2\end{array}\right)+1$.

Lemma 1. [10, Lemma 1] For every $\pi, \epsilon_{n} \in S_{n}$,

$$
d_{K}\left(\epsilon_{n}, \pi\right)+d_{K}\left(\epsilon_{n}, \pi^{r}\right)=w_{K}(\pi)+w_{K}\left(\pi^{r}\right)=d_{K}\left(\pi, \pi^{r}\right)=\left(\begin{array}{l}
n \\
2
\end{array}\right) .
$$

By Lemma 1, we can obtain the following lemma.

Lemma 2. For any $0 \leq i \leq\left\lfloor\frac{\left(\begin{array}{c}n \\ 2\end{array}\right)}{2}\right\rfloor$,

$$
S_{K}^{n}(i)=S_{K}^{n}\left(\left(\begin{array}{l}
n \\
2
\end{array}\right)-i\right)
$$

Proof. Let $m=\left(\begin{array}{l}n \\ 2\end{array}\right)$. We just need to prove that $\left|S_{K}^{n}\left(\epsilon_{n}, i\right)\right|=\left|S_{K}^{n}\left(\epsilon_{n}, m-i\right)\right|$. First we define a function $f: S_{K}^{n}\left(\epsilon_{n}, i\right) \rightarrow S_{K}^{n}\left(\epsilon_{n}, m-i\right)$, where $f(\pi)=\pi^{r}$ for any $\pi \in S_{K}^{n}\left(\epsilon_{n}, i\right)$.

If $\pi \in S_{K}^{n}\left(\epsilon_{n}, i\right)$, then $w_{K}(\pi)=i$. By $(1), w_{K}\left(\pi^{r}\right)=\left(\begin{array}{c}n \\ 2\end{array}\right)-i=m-i$. Hence, $f(\pi) \in S_{K}^{n}\left(\epsilon_{n}, m-i\right)$. Moreover, we can easily prove that the function $f$ is reasonable and bijection. Thus, $S_{K}^{n}(i)=S_{K}^{n}\left(\left(\begin{array}{l}n \\ 2\end{array}\right)-i\right)$.

When $i=0$ or $1, S_{K}^{n}(0)=1$ and $S_{K}^{n}(1)=n-1$. We will further give a recursive formula of $S_{K}^{n}(r)$ in the following lemma.

Lemma 3. For all $4 \leq n$ and $2 \leq i \leq n-1$,

$$
S_{K}^{n}(i)=\sum_{j=0}^{i} S_{K}^{n-1}(j) .
$$

Moreover, for all $5 \leq n$ and $n \leq i \leq\left\lfloor\frac{\left(\begin{array}{c}n \\ 2\end{array}\right)}{2}\right\rfloor$,

$$
S_{K}^{n}(i)=\sum_{j=i-(n-1)}^{i} S_{K}^{n-1}(j) .
$$

Proof. When $4 \leq n$ and $2 \leq i \leq n-1$, we define $S_{K}^{n}\left(\epsilon_{n}, i, j\right) \triangleq\left\{\pi \in S_{K}^{n}\left(\epsilon_{n}, i\right) \mid \pi(j)=n\right\}$ for $n-i \leq j \leq n$, i.e., $\pi \in S_{K}^{n}\left(\epsilon_{n}, i\right)$ is an element of $S_{K}^{n}\left(\epsilon_{n}, i, j\right)$ if $n$ appears at the $j$ th position of $\pi$. For $\pi \in S_{K}^{n}\left(\epsilon_{n}, i\right)$, the number of inversions in the permutation $\pi$ is $i$. If $\pi(j)=n,(\pi(k), n)$ is an inversion for all $j+1 \leq k \leq n$. Hence, for any $\pi \in S_{K}^{n}\left(\epsilon_{n}, i\right)$, $n$ can only appear at the $j$ th position of $\pi$ for every $n-i \leq j \leq n$. So, we obtain that $S_{K}^{n}\left(\epsilon_{n}, i\right)=\cup_{j=n-i}^{n} S_{K}^{n}\left(\epsilon_{n}, i, j\right)$.

For all $n-i \leq j \leq n$, we define $f_{j}: S_{K}^{n}\left(\epsilon_{n}, i, j\right) \rightarrow S_{K}^{n-1}\left(\epsilon_{n-1}, i-(n-j)\right)$, where $f_{j}(\pi)=$ $[\pi(1), \pi(2), \ldots, \pi(j-1), \pi(j+1), \ldots, \pi(n)]$ for any $\pi \in S_{K}^{n}\left(\epsilon_{n}, i, j\right)$. That is, we delete the element $n$ of $\pi$ to obtain $f_{j}(\pi)$. Obviously, $f_{j}$ is injective. For $\pi_{1} \in S_{K}^{n-1}\left(\epsilon_{n-1}, i-(n-j)\right)$, 
we define $\pi$ such that $\pi(k)=\pi_{1}(k)$ for $1 \leq k \leq j-1, \pi(j)=n$, and $\pi(k)=\pi_{1}(k-1)$ for $j+1 \leq k \leq n$. Then, $\pi \in S_{n}$ and $w_{K}(\pi)=w_{K}\left(\pi_{1}\right)+(n-j)=i$. Thus, $\pi \in S_{K}^{n}\left(\epsilon_{n}, i, j\right)$ and $f_{j}(\pi)=\pi_{1}$. So, we obtain that $f_{j}$ is bijection for all $n-i \leq j \leq n$.

Since all the set $S_{K}^{n}\left(\epsilon_{n}, i, j\right)$ are pairwise disjoint and all the $f_{j}$ are bijection for all $n-i \leq j \leq n$, we have

$$
\begin{aligned}
S_{K}^{n}(i) & =\left|S_{K}^{n}\left(\epsilon_{n}, i\right)\right|=\left|\cup_{j=n-i}^{n} S_{K}^{n}\left(\epsilon_{n}, i, j\right)\right|=\sum_{j=n-i}^{n}\left|S_{K}^{n}\left(\epsilon_{n}, i, j\right)\right| \\
& =\sum_{j=n-i}^{n}\left|S_{K}^{n-1}\left(\epsilon_{n-1}, i-(n-j)\right)\right|=\sum_{j=0}^{i} S_{K}^{n-1}(j) .
\end{aligned}
$$

Similarly, for all $5 \leq n$ and $n \leq i \leq\left\lfloor\frac{\left(\begin{array}{c}n \\ 2\end{array}\right)}{2}\right\rfloor$, then $\left\lfloor\frac{\left(\begin{array}{c}n \\ 2\end{array}\right)}{2}\right\rfloor \leq\left(\begin{array}{c}n-1 \\ 2\end{array}\right)$. Thus, for all $i-(n-1) \leq$ $j \leq\left\lfloor\frac{\left(\begin{array}{c}n \\ 2\end{array}\right)}{2}\right\rfloor, S_{K}^{n-1}(j)$ exists. So, we also prove that

$$
S_{K}^{n}(i)=\sum_{j=i-(n-1)}^{i} S_{K}^{n-1}(j) .
$$

Furthermore, we give the recursive formula of $S_{K}^{n}(i)$ for all $4 \leq n$ and $4 \leq i \leq n-1$ in the following lemma. For convenience, for any function $f(t)$ and two positive integers $i<t$, we denote $\sum_{l=t}^{i} f(l)=0$.

Lemma 4. For all $4 \leq n$ and $4 \leq i \leq n-1$, there exists a unique integer $t$ such that $\left(\begin{array}{c}t-1 \\ 2\end{array}\right)<i \leq\left(\begin{array}{l}t \\ 2\end{array}\right)$ and $t \geq 4$. Then, we have

$$
S_{K}^{n}(i)=S_{K}^{t}\left(\left(\begin{array}{l}
t \\
2
\end{array}\right)-i\right)+\sum_{l=t}^{i-1} \sum_{j=i-l}^{i-1} S_{K}^{l}(j)+\sum_{l=i}^{n-1} \sum_{j=0}^{i-1} S_{K}^{l}(j) .
$$

Proof. When $4 \leq n$ and $4 \leq i \leq n-1$, by (3), we have

$$
S_{K}^{n}(i)-S_{K}^{n-1}(i)=\sum_{j=0}^{i-1} S_{K}^{n-1}(j) .
$$

In (6), we set $n$ to $i+1, \ldots, n$ and obtain $n-i$ equations, respectively. Then by summing all the equations, we have

$$
S_{K}^{n}(i)-S_{K}^{i}(i)=\sum_{l=i}^{n-1} \sum_{j=0}^{i-1} S_{K}^{l}(j) .
$$

For $j<i$ and $i<n$, if $S_{K}^{n}(j)$ and $S_{K}^{i}(i)$ are known, then by (7) we can compute $S_{K}^{n}(i)$. In the following, we will compute $S_{K}^{i}(i)$. By $(4)$, for $i \leq\left(\begin{array}{c}i-1 \\ 2\end{array}\right)$ (i.e., $\left.4 \leq i\right)$, we obtain that

$$
S_{K}^{i}(i)-S_{K}^{i-1}(i)=\sum_{j=1}^{i-1} S_{K}^{i-1}(j)
$$


For $4 \leq i$, we can find an integer $t$ such that $\left(\begin{array}{c}t-1 \\ 2\end{array}\right)<i \leq\left(\begin{array}{l}t \\ 2\end{array}\right)$ and $t \geq 4$. Then, $\left(\begin{array}{l}t \\ 2\end{array}\right)+$ $\frac{(t-1)(t-4)}{2}<2 i$ and $t<\left(\begin{array}{c}t-1 \\ 2\end{array}\right)$ for $5 \leq t$. When $i=4$, we have $t=4$. When $5 \leq i$, we have $4 \leq t, i \leq\left(\begin{array}{l}t \\ 2\end{array}\right)<2 i$, and $t<i$.

Thus, we obtain

$$
0 \leq\left(\begin{array}{l}
t \\
2
\end{array}\right)-i<i
$$

When $i=4, S_{K}^{4}(4)=S_{K}^{4}\left(\left(\begin{array}{l}4 \\ 2\end{array}\right)-4\right)=S_{K}^{4}(2)$.

Similarly, when $4<i$, in (4), we set $n$ to $t+1, \ldots, i$ and obtain $i-t$ equations, respectively. By summing all the equations, we have

$$
S_{K}^{i}(i)-S_{K}^{t}(i)=\sum_{l=t}^{i-1} \sum_{j=i-l}^{i-1} S_{K}^{l}(j) .
$$

Combining (2), (9), and (10), we have

$$
S_{K}^{i}(i)=S_{K}^{t}\left(\left(\begin{array}{l}
t \\
2
\end{array}\right)-i\right)+\sum_{l=t}^{i-1} \sum_{j=i-l}^{i-1} S_{K}^{l}(j) .
$$

When $4 \leq i$, we also have $S_{K}^{i}(i)=S_{K}^{t}\left(\left(\begin{array}{l}t \\ 2\end{array}\right)-i\right)+\sum_{l=t}^{i-1} \sum_{j=i-l}^{i-1} S_{K}^{l}(j)$. When $i=t=4$, the second term (i.e., $\left.\sum_{l=4}^{3} \sum_{j=i-l}^{i-1} S_{K}^{l}(j)\right)$ is zero. Finally, by $(7)$ and (11), we can obtain the expression of $S_{K}^{n}(i)$ in the above lemma.

Specifically, we give the formulas of $S_{K}^{n}(2)$ and $S_{K}^{n}(3)$ for all $3 \leq n$ as follows.

Lemma 5. For all $3 \leq n$, we have

$$
\begin{gathered}
S_{K}^{n}(2)=\frac{n(n-1)}{2}-1, \\
S_{K}^{n}(3)=\frac{n^{3}-7 n}{6} .
\end{gathered}
$$

Proof. When $i=2$, by (6), we have

$$
S_{K}^{n}(2)-S_{K}^{2}(2)=\sum_{l=2}^{n-1} \sum_{j=0}^{1} S_{K}^{l}(j) .
$$

Since $S_{K}^{n}(0)=1, S_{K}^{n}(1)=n-1$ and $S_{K}^{2}(2)=0$, by (14), we have

$$
S_{K}^{n}(2)=\sum_{l=2}^{n-1} \sum_{j=0}^{1} S_{K}^{l}(j)=\sum_{l=2}^{n-1} l=\frac{n(n-1)}{2}-1 .
$$

Similarly, when $i=3$, by (6), we have

$$
S_{K}^{n}(3)-S_{K}^{3}(3)=\sum_{l=3}^{n-1} \sum_{j=0}^{2} S_{K}^{l}(j) .
$$


Since $S_{K}^{n}(0)=1, S_{K}^{n}(1)=n-1, S_{K}^{n}(2)=\frac{n(n-1)}{2}-1$, and $S_{K}^{3}(3)=1$, by (16), we have

$$
S_{K}^{n}(3)=S_{K}^{3}(3)+\sum_{l=3}^{n-1} \sum_{j=0}^{2} S_{K}^{l}(j)=1+\sum_{l=3}^{n-1} \frac{l^{2}+l-2}{2}=\frac{n^{3}-7 n}{6} .
$$

According to (15) and (17), we can obtain the expressions of $S_{K}^{n}(2)$ and $S_{K}^{n}(3)$ as (12) and (13), respectively.

Here, we easily obtain $S_{K}^{2}(0)=S_{K}^{2}(1)=1$. By Lemma 5 , when $n=3$, we have $S_{K}^{3}(0)=1, S_{K}^{3}(1)=2, S_{K}^{3}(2)=2$, and $S_{K}^{3}(3)=1$. By Lemma 5 and Lemma 2, we have $S_{K}^{4}(0)=1, S_{K}^{4}(1)=3, S_{K}^{4}(2)=5, S_{K}^{4}(3)=6, S_{K}^{4}(4)=5, S_{K}^{4}(5)=3$, and $S_{K}^{4}(6)=1$.

If all the $S_{K}^{n}(j)$ for all $n$ and $j \leq i-1$ are known, by Lemma 4 , we can compute $S_{K}^{n}(i)$ for $4 \leq n$ and $4 \leq i \leq n-1$. Next we present an example to compute $S_{K}^{n}(i)$ in Lemma 4 .

Example 1. When $i=4,\left(\begin{array}{l}3 \\ 2\end{array}\right)<4 \leq\left(\begin{array}{l}4 \\ 2\end{array}\right)$. Then, we obtain $t=4$ in Lemma 4 . Furthermore, by (5), we have

$$
S_{K}^{n}(4)=S_{K}^{4}\left(\left(\begin{array}{l}
4 \\
2
\end{array}\right)-4\right)+\sum_{l=4}^{3} \sum_{j=i-l}^{i-1} S_{K}^{l}(j)+\sum_{l=4}^{n-1} \sum_{j=0}^{3} S_{K}^{l}(j) .
$$

By Lemma 5, we have $S_{K}^{4}\left(\left(\begin{array}{l}4 \\ 2\end{array}\right)-4\right)=S_{K}^{4}(2)=5$. Thus,

$$
S_{K}^{n}(4)=5+\sum_{l=4}^{n-1}\left(1+(l-1)+\frac{l(l-1)}{2}-1+\frac{l^{3}-7 l}{6}\right)=\frac{n(n+1)\left(n^{2}+n-14\right)}{24} .
$$

In the following, we give the recursive formula of $S_{K}^{n}(i)$ for all $5 \leq n$ and $n \leq i \leq\left\lfloor\frac{\left(\begin{array}{l}n \\ 2\end{array}\right)}{2}\right\rfloor$.

Lemma 6. For all $5 \leq n$ and $n \leq i \leq\left\lfloor\frac{\left(\begin{array}{c}n \\ 2\end{array}\right)}{2}\right\rfloor$, there exists a unique integer $t$ such that $\left(\begin{array}{c}t-1 \\ 2\end{array}\right)<i \leq\left(\begin{array}{l}t \\ 2\end{array}\right)$ and $t \geq 4$. Then, we have

$$
S_{K}^{n}(i)=S_{K}^{t}\left(\left(\begin{array}{l}
t \\
2
\end{array}\right)-i\right)+\sum_{l=t}^{i-1} \sum_{j=i-l}^{i-1} S_{K}^{l}(j)-\sum_{l=n}^{i-1} \sum_{j=i-l}^{i-1} S_{K}^{l}(j) .
$$

Proof. When $5 \leq n$ and $n \leq i \leq\left\lfloor\frac{\left(\begin{array}{c}n \\ 2\end{array}\right)}{2}\right\rfloor$, in (4), we set $n$ to $n+1, \ldots, i$, respectively. Then we obtain $n-i$ equations and sum all the equations. Thus, we have

$$
S_{K}^{i}(i)-S_{K}^{n}(i)=\sum_{l=n}^{i-1} \sum_{j=i-l}^{i-1} S_{K}^{l}(j)
$$

By (11) and (20), we have

$$
S_{K}^{n}(i)=S_{K}^{t}\left(\left(\begin{array}{l}
t \\
2
\end{array}\right)-i\right)+\sum_{l=t}^{i-1} \sum_{j=i-l}^{i-1} S_{K}^{l}(j)-\sum_{l=n}^{i-1} \sum_{j=i-l}^{i-1} S_{K}^{l}(j) .
$$

When $i=n$, the third term (i.e., $\left.\sum_{l=n}^{i-1} \sum_{j=i-l}^{i-1} S_{K}^{l}(j)\right)$ is zero. 
Example 2. When $i=5$ and $n=5$, we have $\left(\begin{array}{l}3 \\ 2\end{array}\right)<5 \leq\left(\begin{array}{l}4 \\ 2\end{array}\right)$. Then, we obtain $t=4$ in Lemma 6. Furthermore, by (19), we have

$$
S_{K}^{5}(5)=S_{K}^{4}\left(\left(\begin{array}{l}
4 \\
2
\end{array}\right)-5\right)+\sum_{l=4}^{4} \sum_{j=5-l}^{4} S_{K}^{l}(j) .
$$

Thus, $S_{K}^{5}(5)=S_{K}^{4}(1)+\sum_{j=1}^{4} S_{K}^{4}(j)=3+(3+5+6+5)=22$.

For every $6 \leq n$, due to $i=5 \leq n-1, S_{K}^{n}(5)$ can be computed by Lemma 4 .

Hence, if $S_{K}^{n}(j)$ are known for all $1 \leq j \leq i-1$ and $n$, we will compute $S_{K}^{n}(i)$ for all $n$ in the next two steps. For $5 \leq i$, there exists a unique integer $t$ such that $\left(\begin{array}{c}t-1 \\ 2\end{array}\right)<i \leq\left(\begin{array}{l}t \\ 2\end{array}\right)$. Then, for every $2 \leq l \leq t-1, S_{K}^{l}(i)=0$. First, when $t \leq l \leq i$, if $i>\left\lfloor\frac{\left(\begin{array}{l}l \\ 2\end{array}\right)}{2}\right\rfloor$, we have $S_{K}^{l}(i)=S_{K}^{l}\left(\left(\begin{array}{l}l \\ 2\end{array}\right)-i\right)$ where $\left(\begin{array}{l}l \\ 2\end{array}\right)-i<i$; otherwise, by Lemma 6 , we compute $S_{K}^{l}(i)$ for $i \leq\left\lfloor\frac{\left(\begin{array}{c}l \\ 2\end{array}\right)}{2}\right\rfloor$. Second, when $i+1 \leq l$, we compute $S_{K}^{l}(i)$ by Lemma 4 .

When $i=5$, we can compute $S_{K}^{n}(5)$ for all $n$. Here, $t=4$. Then, $S_{K}^{4}(5)=S_{K}^{4}\left(\left(\begin{array}{l}4 \\ 2\end{array}\right)-\right.$ $5)=S_{K}^{4}(1)=3$ and $S_{K}^{5}(5)=22$ by Lemma 6 in Example 2 . In the following, we will give the formula of $S_{K}^{n}(5)$ for all $6 \leq n$ by Lemma 4 .

Example 3. When $i=5$ and $6 \leq n$, by Lemma 4 and (7), we have

$$
S_{K}^{n}(5)=S_{K}^{5}(5)+\sum_{l=5}^{n-1} \sum_{j=0}^{4} S_{K}^{l}(j) .
$$

By Examples 1 and 2 and Lemma 5, we have

$$
\begin{aligned}
S_{K}^{n}(5) & =22+\sum_{l=5}^{n-1}\left(1+(l-1)+\frac{l(l-1)}{2}-1+\frac{l^{3}-7 l}{6}+\frac{l(l+1)\left(l^{2}+l-14\right)}{24}\right) \\
& =\frac{(n-1)\left(n^{4}+6 n^{3}-9 n^{2}-74 n-120\right)}{120}
\end{aligned}
$$

for all $5 \leq n$.

By Lemmas 2, 4, and 6, we can obtain the property of $S_{K}^{n}(i)$ for all $6 \leq i$ and $n$ as follows.

Proposition 2. When $6 \leq i$, we can compute $S_{K}^{n}(i)$ for all $5 \leq n$ by using Lemmas 2 , 4 , and 6.

Proof. For all $0 \leq i \leq 5$ and $3 \leq n$, all the $S_{K}^{n}(i)$ are computed. We can compute $S_{K}^{n}(i)$ for all $n$ by using $S_{K}^{n}(j)$ for all $1 \leq j \leq i-1$ and $n$.

First, we find an integer $t$ such that $\left(\begin{array}{c}t-1 \\ 2\end{array}\right)<i \leq\left(\begin{array}{c}t \\ 2\end{array}\right)$. For every $t \leq l \leq i$, if $i>\left\lfloor\frac{\left(\begin{array}{c}l \\ 2\end{array}\right)}{2}\right\rfloor$, we have $S_{K}^{l}(i)=S_{K}^{l}\left(\left(\begin{array}{l}l \\ 2\end{array}\right)-i\right)$ where $\left(\begin{array}{l}l \\ 2\end{array}\right)-i<i$; else if $i \leq\left\lfloor\frac{\left(\begin{array}{l}l \\ 2\end{array}\right)}{2}\right\rfloor$, we compute $S_{K}^{l}(i)$ by Lemma 6 . Second, for every $i+1 \leq l$, we compute $S_{K}^{l}(i)$ by Lemma 4 . So, we can obtain $S_{K}^{n}(i)$ for all $5 \leq n$ and $6 \leq i$. 


\subsection{The size of a ball of radius $r$ in $S_{n}$ under the Kendall $\tau$-metric}

In this subsection, we will give the size of a ball with radius $r$ in $S_{n}$ under the Kendall $\tau$ metric and give recursive formula of $B_{K}^{n}(r)$ by using $S_{K}^{n}(r)$. We easily obtain the following lemma about the relationship between $B_{K}^{n}(r)$ and $S_{K}^{n}(r)$.

Lemma 7. For any $0 \leq r \leq\left(\begin{array}{l}n \\ 2\end{array}\right)$, we have

$$
B_{K}^{n}(r)=\sum_{l=0}^{r} S_{K}^{n}(l)
$$

Given $S_{K}^{n}(i)$ for all $0 \leq i \leq r-1$, by Lemmas 4,6 and 7 , we easily obtain the recursion formula of $B_{K}^{n}(r)$ in the following theorem.

Theorem 1. Suppose $S_{K}^{n}(i)$ are known for all $0 \leq i \leq r-1$ and $5 \leq n$. If $4 \leq r \leq\left\lfloor\frac{\left(\begin{array}{l}n \\ 2\end{array}\right)}{2}\right\rfloor$, there exists a unique integer $t$ such that $\left(\begin{array}{c}t-1 \\ 2\end{array}\right)<r \leq\left(\begin{array}{c}t \\ 2\end{array}\right)$. When $4 \leq r \leq n-1$, we have

$$
B_{K}^{n}(r)=\sum_{l=0}^{r-1} S_{K}^{n}(l)+S_{K}^{t}\left(\left(\begin{array}{l}
t \\
2
\end{array}\right)-r\right)+\sum_{l=t}^{r-1} \sum_{j=r-l}^{r-1} S_{K}^{l}(j)+\sum_{l=r}^{n-1} \sum_{j=0}^{r-1} S_{K}^{l}(j) .
$$

When $n \leq r \leq\left\lfloor\frac{\left(\begin{array}{l}n \\ 2\end{array}\right)}{2}\right\rfloor$, we have

$$
B_{K}^{n}(r)=\sum_{l=0}^{r-1} S_{K}^{n}(l)+S_{K}^{t}\left(\left(\begin{array}{l}
t \\
2
\end{array}\right)-r\right)+\sum_{l=t}^{r-1} \sum_{j=r-l}^{r-1} S_{K}^{l}(j)-\sum_{l=n}^{r-1} \sum_{j=r-l}^{r-1} S_{K}^{l}(j) .
$$

Specially, we have $B_{K}^{n}(0)=1$ and $B_{K}^{n}(1)=n$. When $r=2$, for all $n \geq 2$, we have

$$
B_{K}^{n}(2)=\sum_{l=0}^{2} S_{K}^{n}(l)=\left(1+n-1+\frac{n(n-1)}{2}-1\right)=\frac{(n+2)(n-1)}{2} .
$$

When $r=3$, for all $n \geq 3$, we have

$$
B_{K}^{n}(3)=\sum_{l=0}^{3} S_{K}^{n}(l)=\left(1+n-1+\frac{n(n-1)}{2}-1+\frac{n^{3}-7 n}{6}\right)=\frac{(n+1)\left(n^{2}+2 n-6\right)}{6} .
$$

Example 4. When $r=4$ and $4 \leq n$, by Example 1 and Theorem 1, we have

$$
\begin{aligned}
B_{K}^{n}(4) & =\sum_{l=0}^{3} S_{K}^{n}(l)+S_{K}^{4}\left(\left(\begin{array}{l}
4 \\
2
\end{array}\right)-4\right)+\sum_{l=4}^{3} \sum_{j=4-l}^{4-1} S_{K}^{l}(j)+\sum_{l=4}^{n-1} \sum_{j=0}^{3} S_{K}^{l}(j) \\
& =\frac{(n+2)(n+1)\left(n^{2}+3 n-12\right)}{24} .
\end{aligned}
$$


Moreover, when $r=5$ and $5 \leq n$, by Example 3 and Theorem 1, we have

$$
\begin{aligned}
B_{K}^{n}(5) & =\sum_{l=0}^{4} S_{K}^{n}(l)+S_{K}^{n}(5) \\
& =\frac{(n+7) n\left(n^{3}+3 n^{2}-6 n-28\right)}{120} .
\end{aligned}
$$

When $r \geq 6$, we can compute $B_{K}^{n}(r)$ by using Proposition 2 and Theorem 1 .

\section{The nonexistence of a perfect $t$-error-correcting code in $S_{n}$ under the Kendall $\tau$-metric for some $n$ and $t=2,3,4$, or 5}

In this section, we will prove the nonexistence of a perfect $t$-error-correcting code in $S_{n}$ under the Kendall $\tau$-metric for some $n$ and $t=2,3,4$, or 5 by using the sphere-packing upper bound. By Proposition 1, we give the necessary condition of the existence of a perfect $t$-error-correcting code in $S_{n}$ under the Kendall $\tau$-metric.

Lemma 8. For any $0 \leq t \leq\left(\begin{array}{l}n \\ 2\end{array}\right)$, if there exists one perfect $t$-error-correcting code $C$ in $S_{n}$ under the Kendall $\tau$-metric. Then, we must have

$$
B_{K}^{n}(t) \cdot|C|=n ! .
$$

That is, the necessary condition of the existence of a perfect $t$-error-correcting code in $S_{n}$ under the Kendall $\tau$-metric is $B_{K}^{n}(t) \mid n$ !.

Proof. By the sphere-packing upper bound in Proposition 1, if there exists one perfect $t$-error-correcting code $C$ in $S_{n}$ under the Kendall $\tau$-metric, we must have $B_{K}^{n}(t) \cdot|C|=n$ !. Thus, $B_{K}^{n}(t) \mid n$ !. So, the necessary condition of the existence of a perfect $t$-error-correcting code in $S_{n}$ under the Kendall $\tau$-metric is $B_{K}^{n}(t) \mid n !$.

According to Lemma 8, we have the following theorem which illustrate the nonexistence of a perfect $t$-error-correcting code in $S_{n}$ under the Kendall $\tau$-metric.

Theorem 2. For any $0 \leq t \leq\left(\begin{array}{c}n \\ 2\end{array}\right)$, if $B_{K}^{n}(t)$ has a prime factor $p>n$, then there does not exist one perfect t-error-correcting code in $S_{n}$ under the Kendall $\tau$-metric.

Proof. By Lemma 8, the necessary condition of the existence of a perfect $t$-error-correcting code in $S_{n}$ under the Kendall $\tau$-metric is $B_{K}^{n}(t) \mid n$ !. Since $B_{K}^{n}(t)$ has a prime factor $p>n$, we have $B_{K}^{n}(t) \nmid n$ !. So, we prove the above result.

In the following, we will dicuss the nonexistence of a perfect $t$-error-correcting code in $S_{n}$ for some $n$ and $t=2,3,4$, or 5 by using Theorem 2 .

When $t=2$, by $(25)$, we have $B_{K}^{n}(2)=\frac{(n+2)(n-1)}{2}$. By Theorem 2 , we can prove the nonexistence of a perfect two-error-correcting code in $S_{n}$, where $n+2>6$ is a prime. 
When $t=3$, by $(26)$, we have $B_{K}^{n}(3)=\frac{(n+1)\left(n^{2}+2 n-6\right)}{6}$. First, if $n+1>6$ is a prime, then $B_{K}^{n}(3)$ have a prime factor $n+1>n$. Second, we compute $n^{2}+2 n-6$ for $4 \leq n \leq 33$ and obtain that $(n+1)\left(n^{2}+2 n-6\right)$ has a prime factor $p>n$ except $n=13$ and $n=26$. If $n=13, B_{K}^{13}(3)=441=9 \times 7^{2}$. Thus, $441 \nmid 13$ !. If $n=26, B_{K}^{26}(3)=3249=9 \times 19^{2}$. Hence, $3249 \nmid 26$ !. So, by Theorem 2 , we can prove the nonexistence of a perfect threeerror-correcting code in $S_{n}$, where $n+1>6$ is a prime, $n^{2}+2 n-6$ has a prime factor $p>n$, or $4 \leq n \leq 33$.

When $t=4$, by $(27)$, we have $B_{K}^{n}(4)=\frac{(n+1)(n+2)\left(n^{2}+3 n-12\right)}{24}$. First, if $n+1>6$ or $n+2>7$ is a prime, then $B_{K}^{n}(3)$ have a prime factor $p>n$. Second, we compute $n^{2}+3 n-12$ for $5 \leq n \leq 19$ and obtain that $\left(n^{2}+3 n-12\right)(n+1)(n+2)$ has a prime factor $p>n$ except $n=13$. If $n=13, B_{K}^{13}(4)=1715=5 \times 7^{3}$. Thus, $1715 \nmid 13$ !. So, by Theorem 2, we can prove the nonexistence of a perfect four-error-correcting code in $S_{n}$, where $n+1>6$ or $n+2>7$ is a prime, $n^{2}+3 n-12$ has a prime factor $p>n$, or $5 \leq n \leq 19$.

When $t=5$, by $(28), B_{K}^{n}(5)=\frac{(n+7) n\left(n^{3}+3 n^{2}-6 n-28\right)}{120}$. By Theorem 2, we can prove the nonexistence of a perfect five-error-correcting code in $S_{n}$, where $n+7 \geq 12$ is a prime or $n^{3}+3 n^{2}-6 n-28$ has a prime factor $p>n$.

By the above discussion, we have the following theorem.

Theorem 3. When $t=2$, there are no perfect two-error-correcting codes in $S_{n}$, where $n+2>6$ is a prime. When $t=3$, there are no perfect three-error-correcting codes in $S_{n}$, where $n+1>6$ is a prime, $n^{2}+2 n-6$ has a prime factor $p>n$, or $4 \leq n \leq 33$. When $t=4$, there are no perfect four-error-correcting codes in $S_{n}$, where $n+1>6$ or $n+2>7$ is a prime, $n^{2}+3 n-12$ has a prime factor $p>n$, or $5 \leq n \leq 19$. When $t=5$, there are no perfect five-error-correcting codes in $S_{n}$, where $n+7 \geq 12$ is a prime or $n^{3}+3 n^{2}-6 n-28$ has a prime factor $p>n$.

\section{Conclusion}

Permutation codes under the Kendall $\tau$-metric have been attracted lots of research interest due to their applications in flash memories. In this paper, we considered the nonexistence of perfect codes under the Kendall $\tau$-metric. We gave the recursive formulas of the size of a ball or a sphere with radius $t$ in $S_{n}$ under the Kendall $\tau$-metric. Specifically, we gave the polynomial expressions of the size of a ball or a sphere with radius $r$ when $t=2,3,4$, or 5 . Finally, we used the sphere-packing upper bound to prove that there are no perfect $t$ error-correcting codes in $S_{n}$ under the Kendall $\tau$-metric for some $n$ and $t=2,3,4$, or 5 . Specifically, we proved that there are no perfect two-error-correcting codes in $S_{n}$, where $n+2>6$ is a prime. We also proved that there are no perfect three-error-correcting codes in $S_{n}$, where $n+1>6$ is a prime, $n^{2}+2 n-6$ has a prime factor $p>n$, or $4 \leq n \leq 33$. We further proved that there are no perfect four-error-correcting codes in $S_{n}$, where $n+1>6$ or $n+2>7$ is a prime, $n^{2}+3 n-12$ has a prime factor $p>n$, or $5 \leq n \leq 19$. We proved that there are no perfect five-error-correcting codes in $S_{n}$, where $n+7 \geq 12$ is a prime or $n^{3}+3 n^{2}-6 n-28$ has a prime factor $p>n$. 


\section{References}

[1] Horvitz M., Etzion T.: Constructions of snake-in-the-box codes for rank modulation. IEEE Trans. Inf. Theory 60, 7016-7025 (2014).

[2] Jiang A., Mateescu R., Schwartz M., Bruck J.: Rank modulation for flash memories. IEEE Trans. Inf. Theory 55, 2659-2673 (2009).

[3] Jiang A., Schwartz M., Bruck J.: Correcting charge-constrained errors in the rankmodulation scheme. IEEE Trans. Inf. Theory 56, 2112-2120 (2010).

[4] Kløve T., Lin T.T., Tsai S.C., Tzeng W.G.: Permutation arrays under the Chebyshev distance. IEEE Trans. Inf. Theory 56, 2611-2617 (2010).

[5] Wang X, Fu F.-W.: On the snake-in-the-box codes for rank modulation under Kendall's $\tau$-metric. Des. Codes Cryptogr. 83, 455-465 (2017).

[6] Wang X, Fu F.-W.: Snake-in-the-box codes under the $\ell_{\infty}$-metric for rank modulation. Des. Codes Cryptogr. 83, 455-465 (2019).

[7] Yehezkeally Y., Schwartz M.: Snake-in-the-box codes for rank modulation. IEEE Trans. Inf. Theory 58, 5471-5483 (2012).

[8] Barg A., Mazumdar A.: Codes in permutations and error correction for rank modulation. IEEE Trans. Inf. Theory 56, 3158-3165 (2010).

[9] Zhang Y.W., Ge G.N.: Snake-in-the-box codes for rank modulation under Kendall's $\tau$-metric. IEEE Trans. Inf. Theory 62, 151-158 (2016).

[10] Buzaglo S., Etzion T.: Bounds on the size of permutation codes with the Kendall $\tau$-metric. IEEE Trans. Inf. Theory 61, 3241-3250 (2015).

[11] Farnoud F., Skachek V., Milenkovic O.: Error-correction in falsh memories via codes in the Ulam metric. IEEE Trans. Inf. Theory 59, 3003-3020 (2013).

[12] Zhou H., Jiang A., Bruck J.: Systematic error-correcting codes for rank modulation. in Proc. IEEE Int. Symp. Inf. Theory, 2978-2982 (2012).

[13] Zhou H., Schwartz M., Jiang A., Bruck J.: Systematic error-correcting codes for rank modulation. IEEE Trans. Inf. Theory 61, 17-32 (2015). 
[14] Wang X, Zhang Y.W., Yang Y.T., Ge G.N.: New bounds of permutation codes under Hamming metric and Kendall's $\tau$-metric. Des. Codes Cryptogr. 85, 533-545 (2017).

[15] Vijayakumaran S.: Largest permutation codes With the Kendall $\tau$-metric in $S_{5}$ and $S_{6}$. IEEE Communications Letters. 20, 1912-1915 (2016). 